\title{
A Method for Diagnosing the State of Electric Actuator Based on Neural Network
}

\author{
Wang Hong-li, Xu Bing, Liu Fu-li, Zheng Yuan \\ (Dept. of 5,Wuhan Mechanical Technology, Wuhan 430075,China) \\ Email:1712178221@qq.com.cn
}

\begin{abstract}
The article, taking neural network (NN) as the tool and electric actuator as study object, proposes a diagnosis method for electric actuator based on self-organization competitive neural network, by which state diagnosis is realized with discrimination capacity of nonlinear dynamic system of neural network as well as comparison of forecast value of the system and measured values of actual parameters. The system incorporated VB6.0 as development tool and SQL Server2000 as backstage database to realize intelligent diagnosis of states with electric actuator.
\end{abstract}

Keywords-State Diagnosis; Electric Actuator; Artificial Intelligence (AI); Neural Network (NN)

\section{Signal Acquisition System Based on Electric Actuator}

In traditional way, certain technical performances of electric actuator are analyzed unexceptionally through directly manual performance test of electric actuator; today, at some industrial workspace where electric actuator is used, most tests are performed by technical personnel based on their experience in combination with theoretical knowledge, which are time-consuming and undesirable in test effects. With increasing application of computer, it's necessary to analyze and test these major performance indicators based on computer aid.

The system acquires position feedback current signal of electric actuator with Keithley2000 digital multi-meter produced by American Keithley, inputs data to the computer via RS-232 serial interfaces of industrial personal computer, realizes acquisition of signal through VB 6.0 MSComm serial communication control programming, then draws curve of acquired signals by MatrixVB for VB software developed by MathWorks for fitting analysis, and then derives performance parameters reflecting system state, so as to realize effective diagnosis Figure 1 is hard wiring diagram of data set, multi-circuit relay switch is connected to industrial personal computer via one ISA card, and then corresponding demand is sent from industrial personal computer to control opening/closing at relay point to realize function of controlling connection/disconnection to current signal source. In order to reduce the interference of additional position feedback at the moment of connection/disconnection to signal source, we've improved circuit connection, i.e., signal at input terminal of servo amplifier from current signal source is not open circuit when disconnecting to signal source, rather, relay switch is closed to cause short circuit, so as to reduce interference by external additional signals on the circuit.

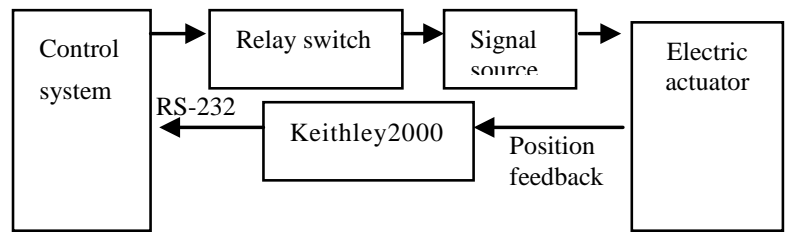

Fig. 1Wiring Diagram for Acquisition of Electric Actuator Signals

II Intelligent Diagnosis Based on Neural Network

\section{A. Network Learning Rules}

Self-organization competitive neural network selected by us is a kind of neural network without teaching of physical teacher and with self-organization function, which, by self-training, can automatically classify input mode and identify current mode from those of detected modes, make most close classification, that is, express classification result by winning competitive neuron nodes.

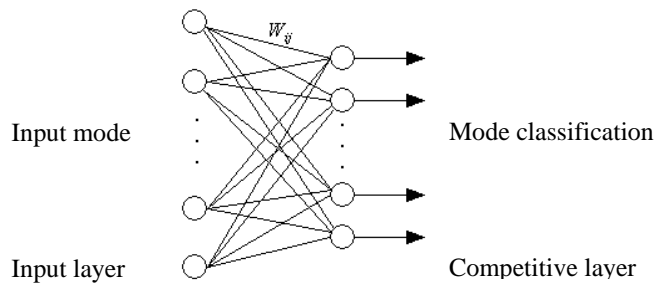

Fig.2 Structure of Basic Competitive Neural Network 


\section{B. Diagnosis of Electric Actuator State}

Base on field experience and related theory, characteristic parameters having impact on operating state of electric actuator are classified, and operating states are subdivided into heathy state, sub-healthy state and morbid state according to combination of these classifications; from the perspective of mode identification, the task of state diagnosis is to identify the state of actuator in operation. If abundant state information is available for the system under study, learning by neural network is feasible, and system state can be mapped with derived parameters.

\section{(1) Principle of System}

To judge the performance of an electric actuator, we need to analyze some of its characteristic parameters. Information on the parameters of electric actuator can be acquired by data acquisition device based on serial communication. Conduct linear least-squares fitting of acquired data, and derive some major characteristic indicators of electric actuator after calculation, such as pure delay time, ascending rate, descending rate, dead band, hysteresis and error.

\section{(2) Set of Electric Actuator States}

Prerequisite of diagnosis is to determine set of electric actuator states, i.e., check common typical states of electric actuator, and find causes of these states, based on practical operation experience and theoretical analysis, common system states of electric actuator are divided into 11 typical modes, expressed as $u_{i}(i=1,2, \cdots 11) ; 18$ signs are extracted, and sign $x_{j}(j=1,2, \cdots 18)$ is defined as follows:

$$
x_{j}=\left\{\begin{array}{l}
1 \\
0
\end{array} \quad, \quad 1=\text { sign exists, } 0=\right.\text { sign non-exists. }
$$

$j=1,2, \cdots, 18$

Base on foregoing classifications of parameters, parameters corresponding to system states are classified; set of typical states of electric actuator is listed in Table 1 , in which the causes of common morbid actuator states (Failure states) are listed, please refer to the manual on repair; corresponding remedies for sub-healthy states are basically consistent with those for morbid states, except for the extent of treatment.

Table 1 Set of Typical Electric Actuator States

\begin{tabular}{|l|l|l|}
\hline$u_{1}$ & Healthy state & All indicators are normal \\
\hline$u_{2}$ & Sub-healthy state & Excess hysteresis \\
\hline$u_{3}$ & Sub-healthy state & Excess pure delay time \\
\hline$u_{4}$ & Sub-healthy state & Excess dead band \\
\hline$u_{5}$ & Sub-healthy state & Excess error \\
\hline$u_{6}$ & Morbid state & Excess hysteresis \\
\hline$u_{7}$ & Morbid state & Excess pure delay time \\
\hline$u_{8}$ & Morbid state & Excess dead band \\
\hline$u_{9}$ & Morbid state & Excess hysteresis \\
\hline$u_{10}$ & Morbid state & Actuator lock-in \\
\hline$u_{11}$ & Morbid state & Inaction \\
\hline
\end{tabular}

\section{(3) Diagnosis of Electric Actuator States}

With set of system states, system diagnosis is available by special means; in most cases, intelligent diagnosis of electric actuator states is realized on the basis of theoretical analysis, in this article, physical object is used as diagnosis model for diagnosing electric actuator states by self-organization competitive neural network of good classification performance introduced above.

Based on the principle of self-organization competitive neural network, first determine input and output vectors of the network; take sign vector $x_{j}(j=1,2, \cdots 18)$ as input, corresponding system state as output, and $X_{j}$ as input of input layer neuron, namely,
18 input nodes (Corresponding to 18 states, respectively) constitute input layer, 11 output nodes (Corresponding to 11 states, respectively) constitute output layer; When output at a given node is 1 , indicating occurrence of corresponding state, connection to external display equipment is available and timely reflection of electric actuator state.

According to the principle of self-organization competitive neural network, carry out VB programming, take learning rate to 0.01 , number of learning processes reflects separation of the sample; practice indicates that, after 2000 times of training, network fully separates various states, corresponding weighed values are 
moderate, for competitive network is simple in structure and algorithm, together with less training time.

Diagnosis can be performed following satisfactory network training, read in weighed values, according to

\section{Software Realization of States Diagnosis}

The software package uses intelligent diagnosis module as the core and database module as backstage, analyzes electric actuator states by two functional modules for data acquisition and curve analysis, and finally realizes diagnosis of electric actuator states.

After drawing of curve by MatrixVB, carry out curve fitting with selected fitting zone, as shown in Figure 3. Yellow line is fitting line, nonlinear curve segment is

\section{IIITest and Evaluation of Program Application}

In order to verify the applicability and reliability of software package, two electric actuators of known states are diagnosed, of which one actuator is in good performance, and the other actuator is in poor sensitivity with excess response delay time; with data acquisition function of the software package, data of both electric actuators are acquired, and the data are entered in database, then express acquired data in physical curve with curve analysis module, and then derive parameters representing electric actuator states through fitting; data curve of healthy electric actuator after fitting is as shown in Figure 3; after analysis, parameters are displayed in "characteristics parameters of actuator zone" in original

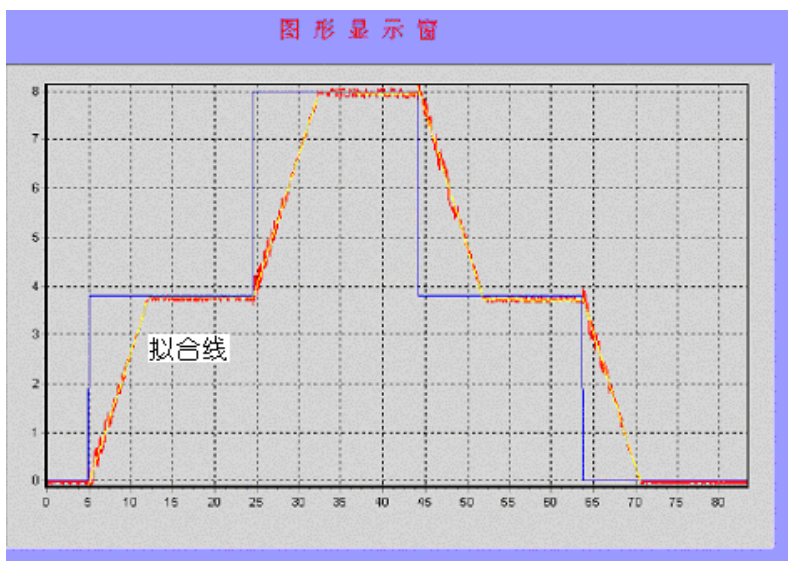

Fig. 3 Curve Fitting Analysis

\section{References:}

[1] Zhou Donghua. Sun Youxian, Technologies for Detecting \& Diagnosing Failures with Control System, Beijing: Tsinghua University Press, 1994

[2] Zhang Yulin, Sun Dongxu, Theories and Applications of Diagnosis of Failures with Dynamic System, Changsha: National University of Defense Technology Press, 1997

[3] Jiao Licheng, Theories of Neural Network System, Xi'an, Xidian University Press, 1992 winning rules of self-organization competitive neural network, 11 common electric actuator states can be identified.

linearized for calculating parameters, that is, a set of parameters representing electric actuator states are derived from curve analysis module, namely, pure delay time, ascending rate, descending rate, dead band, hysteresis and error mentioned above; put these parameters in intelligent diagnosis module for diagnosis by self-organization competitive neural network, so as to identify current actuator state, and display the result on operator interface.

operator interface, and diagnosis results are displayed in "state analysis zone", as shown in Figure 4; likewise, another actuator is diagnosed as in sub-healthy state with excess delay time, and corresponding solution can be found by referring to electric actuator manual.

This suggests that said software is able to diagnose without error that state of electric actuator and gives state parameters, thus it's practically valuable; during software testing, multiple electric actuators are diagnosed for many times, and a lot of failure data are manually made according to theory as test specimen; tests suggest reliable and effective results of diagnosis, and realization of original assumption and applied function.

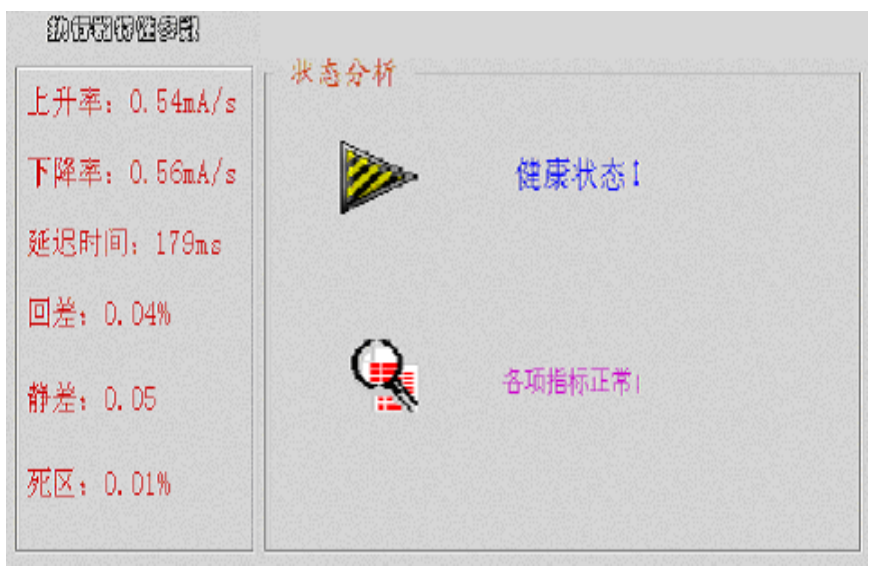

Fig. 4 Interface of Diagnosis Results

[4] Li Hong and Wu Yiding, A Methodological Study on Fault Diagnosis of Aero-engine by ART1 Neural Network [J], Proceedings of the Chinese Society of Universities for Electric Power System and Automation, 2003.

[5] Cong Shuang, Theory and Application of MATLAB Toolbox Oriented Neural Network ( $2^{\text {nd }}$ Edition) $[\mathrm{M}]$, Press of University of Science and Technology of Chin, Hefei, May 2003

[6] Li Renhou, Intelligent Control Theory \& Method [M], ; Xi'an: Xi'an University of Electronic Science and Technology Press, Oct 1999 